\title{
1982-2012年中亚植被变化及其对气候变化的响应
}

\author{
张 琪 ${ }^{1,2}$ 袁秀亮 ${ }^{1,2}$ 陈 䂀 ${ }^{1}$ 罗格平 ${ }^{1}$ 李龙辉 $^{*}$
}

${ }^{1}$ 中国科学院新疆生态与地理研究所, 荒漠与绿洲生态国家重点实验室, 乌鲁木齐 $830011 ;{ }^{2}$ 中国科学院大学, 北京 100049

\begin{abstract}
摘 要 归一化植被指数 $(N D V I)$ 能够反映植被生长状况，被广泛应用于区域乃至全球的植被变化研究中。该文利用 1982-2012年GIMMS-NDVI数据, 通过基于像元的线性趋势分析、偏相关分析，基于场域的经验正交分解(EOF)、奇异值分解 (SVD), 综合时间和空间两个维度上的信息, 研究了近 31 年来中亚植被的变化及其变化中的区域差异, 分析了植被对气候变 化的响应关系。线性趋势分析发现, 34\%的中亚植被 $N D V I$ 显著增长 $(p<0.05)$, 山区植被 $N D V I$ 的增长速率可达到每年 0.004 。 偏相关分析表明, $63 \%$ 的中亚植被受到降水的显著影响 $(p<0.05$, 仅 $4 \%$ 为负相关), 而 $32 \%$ 的植被受到气温的显著影响 $(p<$ 0.05 , 仅 $9 \%$ 为正相关)。EOF分析发现，中亚植被 $N D V I$ 的变化表现出较大的空间差异：山区及东北部的植被 $N D V I$ 变化主要分 为3个阶段, 即先增长(1982-1994年)、后波动(1994-2002年)、然后继续增长(2002-2012年); 而西北部平原区的植被 $N D V I$ 变化 主要表现为两个阶段，即先增长(1982-1994年)而后减少(1994-2012年)。SVD分析表明：1982-2012年间中亚植被受到降水和 气温的共同影响，植被 $N D V I$ 的空间变化特征与降水的空间变化特征较为一致，但西北部和山区的植被 $N D V I$ 对气温的响应存 在差异。
\end{abstract}

关键词 中亚; 气候变化; 归一化植被指数 (NDVI); 经验正交分解(EOF); 奇异值分解(SVD)

引用格式: 张琪, 袁秀亮, 陈㬢, 罗格平, 李龙辉 (2016). 1982-2012年中亚植被变化及其对气候变化的响应. 植物生态学报, 40, 13-23. doi: $10.17521 /$ cjpe.2015.0236

\section{Vegetation change and its response to climate change in Central Asia from 1982 to 2012}

ZHANG Qi ${ }^{1,2}$, YUAN Xiu-Liang ${ }^{1,2}, \mathrm{CHEN} \mathrm{Xi}^{1}$, LUO Ge-Ping ${ }^{1}$, and LI Long-Hui ${ }^{{ }^{*}}$

${ }^{1}$ State Key Laboratory of Desert and Oasis Ecology, Xinjiang Institute of Ecology and Geography, Chinese Academy of Sciences, Ürümqi 830011, China; and ${ }^{2}$ University of Chinese Academy of Sciences, Beijing 100049, China

\section{Abstract}

Aims Central Asia is one of the most vulnerable and sensitive areas to the change in climate. To understand the response of Central Asia ecosystems to climate change, it is important to improve our understanding of vegetation change and its response to climatic variations. Our objective is to explore and analyze the normalized difference vegetation index $(N D V I)$ and its response to climate change in Central Asia during the period 1982-2012.

Methods The linear regression, the empirical orthogonal function (EOF), the singular value decomposition (SVD) and the partial correlation analysis were used to analyze the NDVI change and its response to climate factors in Central Asia during the period of 1982-2012.

Important findings $34 \%$ of vegetation in Central Asia showed a pronounced change in NDVI with a significant trend of increase $(p<0.05)$ and the rate of increase in NDVI exceeded 0.004 per year for mountainous regions. Both air temperature and precipitation showed significant effects on NDVI. Based on partial correlation analysis, $63 \%$ of vegetation was found to be significantly affected by precipitation $(p<0.05)$ while $32 \%$ vegetation was affected by air temperature $(p<0.05)$. The NDVI changes showed increasing trend from 1982 to 1994, fluctuations between 1994 and 2002, and increasing trend again from 2002 to 2012 in mountainous and northeastern areas. While the NDVI changes experienced increasing trend from 1982 to 1994 but decreasing trend from 1994 to 2012 in northwestern areas. Based on the analysis of SVD, the spatial patterns of NDVI variations were consistent with the spatial patterns of precipitation variations. However, the temperature responses of vegetation NDVI differed across the northeast and the mountainous regions in Central Asia.

Key words Central Asia; climate change; normalized difference vegetation index (NDVI); empirical orthogonal function (EOF); singular value decomposition (SVD)

Citation: Zhang Q, Yuan XL, Chen X, Luo GP, Li LH (2016). Vegetation change and its response to climate change in Central Asia from 1982 to 2012. Chinese Journal of Plant Ecology, 40, 13-23. doi: 10.17521/cjpe.2015.0236

收稿日期Received: 2015-06-21 接受日期Accepted: 2015-11-21

* 通信作者Author for correspondence (E-mail: lhli@ms.xjb.ac.cn) 
植被作为陆地生态系统的主体, 通过能量传输 和物质转变联系土壤、大气和水分, 能够反映并表 征气候变化。在全球变化的背景下, 研究植被变化 及其对气候变化的响应已经成为研究全球变化与陆 地生态系统关系中的重要内容(IGBP, 1997)。但植被 变化的监测方式因研究时空尺度的不同而存在很大 的差异。近 30 年, 遥感以重复周期短、覆盖范围广 的优势日渐成为监测植被变化的主要技术手段 (Fensholt et al., 2012)。在遥感获取的多种植被指数 中, 归一化植被指数 $(N D V I)$ 以其明确的物理意义和 简洁的反演算法, 被广泛应用于区域乃至全球植被 变化的研究(Piao et al., 2011)。因此, 以NDVI为表征, 研究植被的变化趋势, 对揭示陆地生态系统的演变 过程具有重要的意义。

气候因子是驱动植被变化的主要因素, 尤其是 气温和降水, 对植被的生长、分布及其碳收支功能 有着重要的影响。中亚是干旱区、半干旱区分布较 集中的地区, 生态系统脆弱, 对气候变化非常敏感 (Lioubimtseva \& Henebry, 2009)。中亚的气温、降水 在过去几十年间发生了显著的变化。基于站点气象 数据和再分析气候数据(Wright et al., 2009; Hu et al., 2014)以及气候模式的模拟结果(Lioubimtseva et al., 2005)均表明中亚区域气温呈现增加趋势。中亚地区 降水表现出较大的年际波动和空间差异性, 但整个 中亚未来的降水总量可能趋于减少(Lioubimtseva \& Henebry, 2009)。此外, 人类活动如开剭、弃耕等也 影响植被类型和植被分布(de Beurs \& Henebry, 2004; de Beurs et al., 2009)。在气候变化和人类活动 的双重影响下, 研究中亚植被变化及其对气候变化 的响应机制, 对进一步研究中亚干旱区、半干旱区 陆地生态系统演变过程有着重要的意义。

在中亚区域, 植被与气候变化的相关研究仍十 分有限。已有研究主要集中在中亚的哈萨克斯坦(de Beurs et al., 2009; Piao et al., 2011), 部分研究探讨 了气候变化下整个中亚植被与气候因子之间的关系, 但其中大多数研究都聚焦于 21 世纪初期以前的植被 变化状况(Propastin et al., 2008a, 2008b)。研究主要 应用的方法是基于像元针对整个时间序列的线性趋 势分析(Propastin et al., 2008a; Gessner et al., 2013; Mohammat et al., 2013)。但这种分析方法可能会将 时间尺度上的某段有意义的变化平均化, 无法描绘 出年际间的短期变化。并且, 基于像元的分析方法
忽略了像元之间的相互作用，可能会造成分析结果 是以孤立的像元存在。因而, 基于像元侧重于时间 序列整体上升或下降的趋势分析, 不足以详细反映 植被的时空变化信息(Piao et al., 2011)。经验正交分 解(EOF) 是一种分析变量场结构特征, 提取变量场 主要特征量的方法。该方法通过分离出变量场的空 间结构特征及其对应的时间系数, 实现对区域变化 特征的表达。Park和Sohn (2010)、张学珍(2014)分 别应用EOF辨识1982-2010年东亚地区 $N D V I$ 年际变 化、1982-2014年北半球中纬度地区秋季 $N D V$ I变化 的主要特征和区域差异。相比基于像元的时间序列 分析, EOF考虑了像元间的相互作用, 并描述了区 域植被变化的时间特征。在对中亚植被的研究中引 入EOF, 用以获取植被变化的区域特征, 实现在空 间、时间两个维度上对植被变化进行更为细致的探 讨。奇异值分解(SVD) 是一种基于两个变量的协方 差矩阵, 获取两变量场相关信息的方法。Prohaska (1976) 首次将SVD引入气象和气候研究, 而后逐渐 应用于植被和气候变化的研究中。SVD通常与EOF 相结合, 用于分析大尺度植被变化及其对气候变化 的响应(Park \& Sohn, 2010; 张学珍, 2014)。因此, 在研究中亚植被对气候变化的响应时引入 SVD, 进一步分析植被和气候因子在耦合变化下的时空特 征。

本文基于1982-2012年的NDVI，通过线性趋势 分析、偏相关分析, 分析了 31 年间中亚植被 $N D V I$ 的 变化趋势及其对气候因子的响应, 并应用EOF研究 植被变化的主要空间模态和时间演变过程, 探讨中 亚植被的局部变化和短期变化。同时, 应用SVD分 析中亚植被与气候因子在耦合变化中的关系, 并探 讨了其区域差异, 旨在揭示中亚植被对气候变化的 响应机制。

\section{1 材料和方法}

\section{1 研究区域及数据}

中亚一般意义上是指西起里海东至中国西部, 南接阿富汗北抵俄罗斯的亚欧大陆腹地。本研究结 合先前研究(陈睵, 2012)对中亚的定义, 将乌兹别克 斯坦、吉尔吉斯斯坦、土库曼斯坦、塔吉克斯坦、 哈萨克斯坦和中国新疆作为研究区。

NDVI数据采用美国国家航天航空局(NASA)全 球监测与模型研究组(GIMMS)提供的1982-2012年 
空间分辨率为 $8 \mathrm{~km}$, 时间分辨率为 15 天的 $N D V I$ (http://www.gscloud.cn)。GIMMS-NDVI是目前时间 序列最长的 $N D V I$ 数据, 与其他数据相比, 其误差 小、精度较高, 已广泛应用于全球及区域植被变化 的研究中(Slayback et al., 2003)。由于GIMMS-NDVI 仍会受到气溶胶、传感器观测视角等因素的影响, 因而对 $N D V I$ 时间序列进行 S-G滤波(Bojanowski et al., 2009), 使其能够正确反映植被的季节变化规 律。为了避免植被过于稀疏(NDVI受土壤辐射亮度 的影响)和被冰雪覆盖(徐希濡, 2005), 本研究选取 31 年 $N D V I$ 年均值大于 0.1 的像元, 并选择其生长季 (4-10月)的数据加以分析。

气象数据是英国East Anglia大学Climate Research Unit (CRU) 提供的高分辨率全球格点观测数 据(http://www.cgiar-csi.org/), 空间分辨率为 $0.5^{\circ}$, 覆 盖全球陆地, 时间尺度较长(1850年至今)且为月分 辨率。该数据是由气象站点观测资料插值得到的, 在中亚(哈萨克斯坦、吉尔吉斯斯坦、塔吉克斯坦、 乌兹别克斯坦、土库曼斯坦、中国新疆)存在365个 站点(Hu et al., 2014)。本文研究选取1982-2012年每 年的月平均气温数据和前一年生长季结束至次年生 长季结束的累积降水数据进行分析。

\section{2 研究方法}

采用基于像元的线性回归模型(最小二乘法)进 行趋势分析, 在满足显著性水平检验的情况下 $(p<$ $0.05)$, 回归系数为正, 即说明 $N D V I$ 在该研究时间段 内显著增加, 为负则说明 $N D V I$ 在该研究时间段内 显著降低。

偏相关用于分析气温或降水与植被的相关程度, 偏相关系数的绝对值越接近于 1 , 代表变量间的相 关程度越大(Johnson \& Wichern, 2007)。

$\mathrm{EOF}$ 通过线性分解, 用相互正交的特征向量(又 称为空间模态) 和时间系数来描述原变量场, 凸显 原变量的时空变化特征(张学珍, 2014), 在本研究中 用以分析 $N D V I$ 、降水、气温的时空变化特征。假设 研究区的变量 $X$ 有 $m$ 个观测点, 每个观测点有 $n$ 次观 测数据, 则 $X$ 可以表达为矩阵形式(公式(1))。公式(1) 可以分解为空间模态和时间系数的乘积之和(公式 (2)和(3))。

$$
X=\left(\begin{array}{ccc}
x_{11} & \cdots & x_{1 n} \\
\vdots & \ddots & \vdots \\
x_{m 1} & \cdots & x_{m n}
\end{array}\right)
$$

$$
\begin{aligned}
x_{i j} & =\sum_{k=1}^{n} v_{i k} \cdot t_{k j} \\
X & =V \cdot T
\end{aligned}
$$

式中 $x_{i j}$ 代表第 $i$ 个观测点的第 $j$ 次观测值, $v$ 和 $t$ 分别为 空间模态和时间系数, $v_{i k} \cdot t_{k j}$ 是第 $k$ 个空间模态及其 对应的时间系数的乘积。空间模态 $V$ 是EOF输入变量 (变量 $X$ 的协方差矩阵 $C$ ) 所对应的特征向量(公式 (4))。由 $V$ 可计算 $X$ 的时间系数 $T$ (公式(5))。由特征值 ( $\Lambda$ 的对角元素)可计算出空间模态的贡献率, 贡献 率大的空间模态即为变量 $X$ 主要的空间模态(空间分 布结构)。

$$
\begin{aligned}
& C=X \cdot X^{T}=(V \cdot T) \cdot(V \cdot T)^{T}=V \cdot \Lambda \cdot V^{T} \\
& T=V^{T} \cdot X
\end{aligned}
$$

以 $N D V I$ 为例, 方差贡献率越大的空间模态越 能反映 31 年间 $N D V I$ 的空间分布特征; 空间模态的 极值点则反映了 31 年间植被变化的敏感区域(随着 时间的变化，该区域 $N D V I$ 变化幅度最大)。时间系数 是空间模态的权重，描述该空间模态的时间变化信 息，其数值越大，表明对应年份的空间分布特征与 该空间模态的相似程度越高。本文采取特征值误差 范围法来判别EOF分解出的空间模态是否具有物理 意义(魏凤英, 2007)。特征值 $\lambda_{j}$ 的误差范围为 $e_{j}$ (公式 (6)), 当相邻的特征值 $\lambda_{j}, \lambda_{j+1}$ 满足公式(7)时, 可认为 两个特征值所对应的空间模态是有价值的信号。其 中 $n$ 为样本量, 在本文中 $n$ 为 31 。

$$
\begin{aligned}
& e_{j}=\lambda_{j}(2 / n)^{1 / 2} \\
& \lambda_{j}-\lambda_{j+1} \geqslant e_{j}
\end{aligned}
$$

由于EOF局限于研究单变量的时空变化, 并不 能反映两组变量之间的相关关系。NDVI与降水、气 温的相互关系在陆地生态系统与气候变化的研究中 更加重要。因此, 本文通过SVD进一步分析植被与 气温或降水在耦合变化中的空间模态、时间变化。

SVD通过线性分解，利用相互正交的特征向量 (也被称为空间模态)来描述两个变量场的变化信 息。通过SVD分析，可以获取成对变量场之间相关 场的空间模态和时间变化信息(Jordi \& Basterretxea, 2012)。在应用SVD之前, 需要将待分析的变量场 $(X$ 和 $Y$ )进行协方差分析, 得到协方差矩阵 $C$ 。基于 SVD 原理, 矩阵 $C$ 可以表达为公式(8)。

$$
C=U \cdot \Lambda \cdot V^{T}
$$

式中, $U$ 和 $V$ 中的向量是 $C$ 的奇异向量, $\Lambda$ 是对角矩阵, 其对角元素为奇异值, $V^{T}$ 是矩阵 $V$ 的转置。根据奇异 值可以计算出每一对奇异向量所代表的空间模态的 方差贡献率，从而得到对应空间模态的时间系数， 
方差贡献率，从而得到对应空间模态的时间系数， 进而通过分析两个变量时间系数的相关程度, 研究 两个变量空间模态的相关性(魏凤英, 2007)。两个变 量时间系数的相关系数越大, 表明对应的空间模态 的变化越一致。在进行相关分析之前, 需要对两个 变量的时间系数分别进行平稳性检验(单位根), 以 避免由于两个序列“不平稳” (即时间序列含有趋势 项、周期项等)造成假相关(Gimeno et al., 1999)。当 时间序列不存在单位根(即时间序列是平稳的)时, 两者的相关系数能够代表两个变量之间的真实关 系。当时间序列中存在单位根(即时间序列是非平稳) 时, 需要对序列进行差分处理, 在获取平稳的序列 以后再计算相关系数。

\section{2 结果和分析}

\subsection{2-2012年NDVI、降水、气温基于像元的时 空变化分析}

为了解中亚 31 年间植被、降水、气温的空间分 布状况和变化趋势, 本研究基于像元计算 1982-2012年中亚 $N D V I 、$ 降水、气温的年均值、变 化趋势和区域年际变化趋势。

31 年 $N D V I$ 年均值, 在天山、阿勒泰山大于 0.7 (图1A), 在哈萨克丘陵、图尔盖高原地区介于 0.3-0.7之间, 在植被分布的边缘地区小于 0.3 。山区 的年降水量大于 $450 \mathrm{~mm}$, 哈萨克丘陵、图尔盖高原 地区年降水量 $300 \mathrm{~mm}$ 左右(图1B)。山区、帕米尔高 原的年均气温明显低于哈萨克丘陵、图尔盖高原(图 $1 C)$ 。1982-2012年中亚 $N D V I$ 和气温均表现为显著的 增加趋势(图1G,1I), 但降水表现出较大的年际波动, 没有表现出显著的变化趋势(图1H)。

基于像元的线性趋势分析(图1D)发现, 在31年 间, $34 \%$ 的植被 $N D V I$ 显著增加 $(p<0.05)$, 山区的增 加速率可达到 $0.004 /$ 年, 但在西北部(图尔盖高原、 图尔盖洼地)显著下降 $(p<0.05)$ 。中亚降水在帕米尔 高原、山区呈增加趋势(图1E), 在图尔盖洼地呈降 低趋势，显著变化的区域仅占 $2 \%(p<0.05)$ 。然而， 整个中亚 $96 \%$ 的区域气温显著增加 $(p<0.05)$ (图 $1 \mathrm{~F})$ 。

为了进一步了解 $N D V I$ 对降水或气温的响应, 基于像元对1982-2012年 $N D V I$ 与降水、 $N D V I$ 与气温 进行了偏相关分析(图2)。结果表明 $N D V I$ 与降水的偏 相关系数绝大多数为正, 具有较好的空间一致性。
$63 \%$ 的植被与降水呈显著相关 $(p<0.05)$, 其中仅有 $4 \%$ 呈显著负相关 $(p<0.05)$, 主要分布在海拔较高 的山区。NDVI与气温的偏相关系数则呈现出明显的 空间差异。9\%的植被与气温呈显著正相关 $(p<0.05)$, 主要分布于东部海拔较高的区域; $23 \%$ 与气温呈现 负相关 $(p<0.05)$, 主要分布于西部平原区域。

\section{$2.21982-2012$ 年NDVI、降水、气温基于场域的时 空变化分析}

\subsubsection{NDVI、降水、气温的EOF分析}

$N D V I$ 的EOF分析结果表明, 前三个空间模态的 累计方差贡献率为 $48 \%$ (表1), 且通过了特征根误差 范围的检验。第一空间模态(图3A)代表了1982-2012 年植被 $N D V I$ 变化的主要空间结构, 数值在空间上 基本保持均一状态, 山区较高, 与 $N D V I$ 的空间变化 趋势(图1D)相似。对应的时间系数(图3C)反映了该 空间模态的时间变化特征, 表现为增长(1982-1994 年)、波动(1994-2002年)、增长(2002-2012年) 3个阶 段。这与 $N D V I$ 年际趋势分析的结果一致(图 $1 \mathrm{G})$, 也 与北半球植被整体增加的趋势相一致(Slayback et $a l .$, 2003)。第二空间模态(图3B)反映了中亚西北部 (哈萨克丘陵、图尔盖高原)与山区的 $N D V I$ 在数值上 存在差异, 结合对应的时间系数(图3D)表明中亚北 部(哈萨克丘陵、图尔盖高原)先增加(1982-1994年) 后减少(1994-2012年), 与山区差异明显。

降水前三个空间模态的累计方差贡献率为 $58 \%$ (表1)。第一空间模态(图4A)反映了山区降水明显高 于其他区域, 但其对应的时间系数(图4C)呈现较大 的年际波动。该空间模态及其时间变化与降水的空 间变化趋势(图1E)和年际变化(图1H)相一致, 也与 先前在天山、帕米尔高原的降水变化的研究结果相 一致(Chen et al., 2011)。第二空间模态的方差贡献率 为 $15 \%$ (图4B), 纬度差异明显, 极高值出现在帕米 尔高原, 极低值出现在图尔盖洼地。时间系数波动 幅度大, 尤其是在 20 世纪 90 年代, 与此阶段的降水 年际波动变化一致(Chen et al., 2011)。

气温的EOF分析, 前三个空间模态的累计方差贡 献率为 $90 \%$ (表1)。第一空间模态(图5A)占总方差的 $74 \%$, 其数值均大于 0 , 在空间上具有很好一致性, 极 值点出现在图尔盖洼地、图尔盖高原。其对应的时间 系数(图5B)呈增长趋势, 反映出在1982-2012年间气 温呈增加趋势。由于第二、第三空间模态的方差解释 率很低, 分别为 $10 \%$ 和6\%。因此, 1982-2012年间中亚 
气温变化的空间特征以第一特征变量为主。
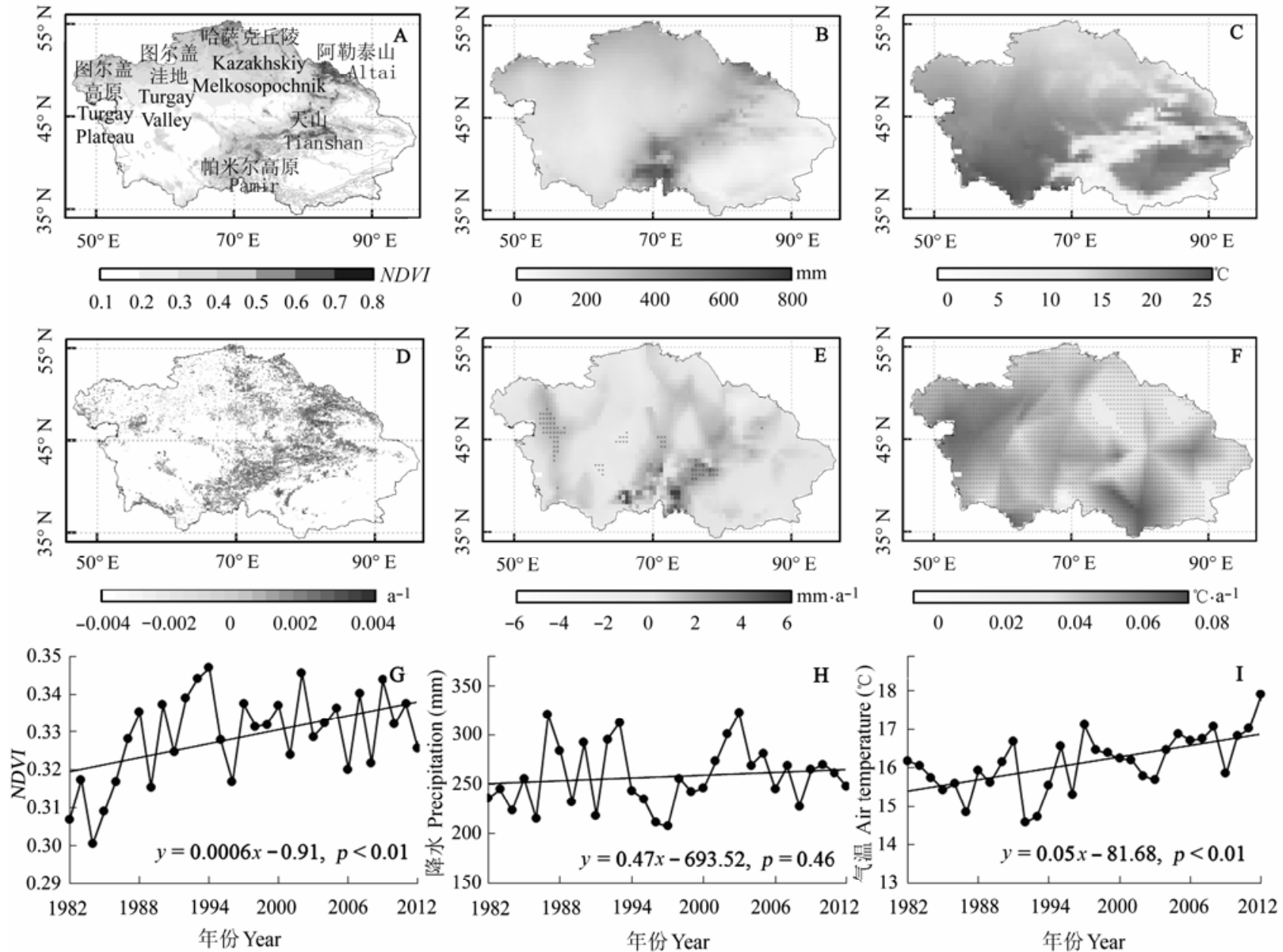

图1 1982-2012年中亚植被年均归一化植被指数 $(N D V I)$ (灰线为等高线, 间距1 $000 \mathrm{~m})(\mathbf{A}) 、$ 年降水量 $(\mathbf{B}) 、$ 年平均气温 $(\mathbf{C})$; 1982-2012年年均 $N D V I(\mathbf{D})(p<0.05)$ 、年降水量 $(\mathbf{E})$ 和年平均气温 $(\mathbf{F})$ 的空间变化趋势(点代表 $p<0.05) ; 1982-2012$ 年年均 $N D V I$ $(\mathbf{G})$ 、年降水量 $(\mathbf{H})$ 和年平均气温 $(\mathbf{I})$ 的年际变化趋势。

Fig. 1 The annual mean of normalized difference vegetation index ( $N D V I)$ (The gray line indicates contour line, the interval between lines is $1000 \mathrm{~m})(\mathbf{A})$, annual precipitation $(\mathbf{B})$, annual mean air temperature (C) in the period of 1982-2012; the spatial pattern of annual mean $N D V I$ change $(\mathbf{D})(p<0.05)$, annual precipitation change $(\mathbf{E})$ and annual mean air temperature change $(\mathbf{F})$ (the point represents $p<0.05)$ in the period of 1982-2012; the temporal trends of the annual mean $N D V I(\mathbf{G})$, the annual precipitation $(\mathbf{H})$, the annual mean air temperature (I) in the period of 1982-2012.

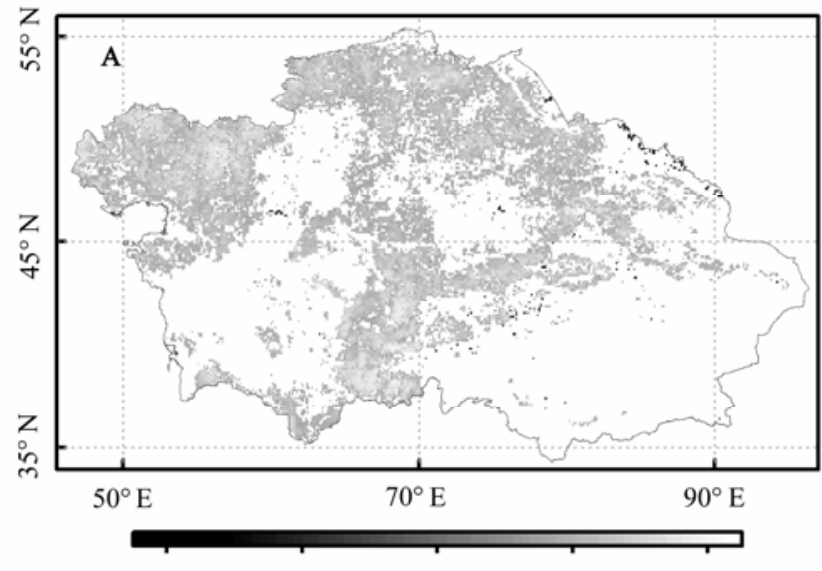

$-0.8$

$-0.4$

0

0.4

0.8

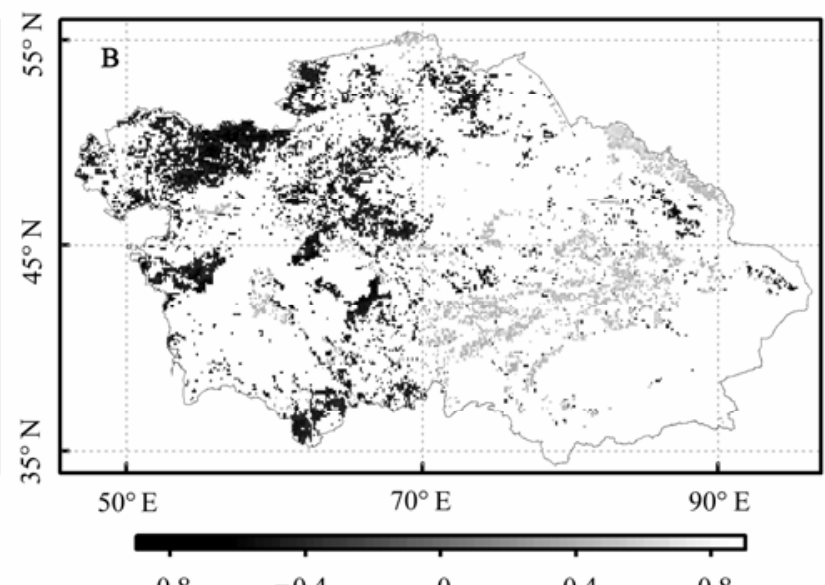

$-0.8$

$-0.4$

0.4

0.8

图2 1982-2012年中亚植被年均归一化植被指数与年降水量 $(\mathbf{A})$ 和年平均气温 $(\mathbf{B})$ 的偏相关系数 $(p<0.05)$ 。

Fig. 2 The partial correlation coefficients between annual mean normalized difference vegetation index (NDVI) and annual precipi- 
tation (A) and annual mean air temperature $(\mathbf{B})$ in the period of 1982-2012 $(p<0.05)$.
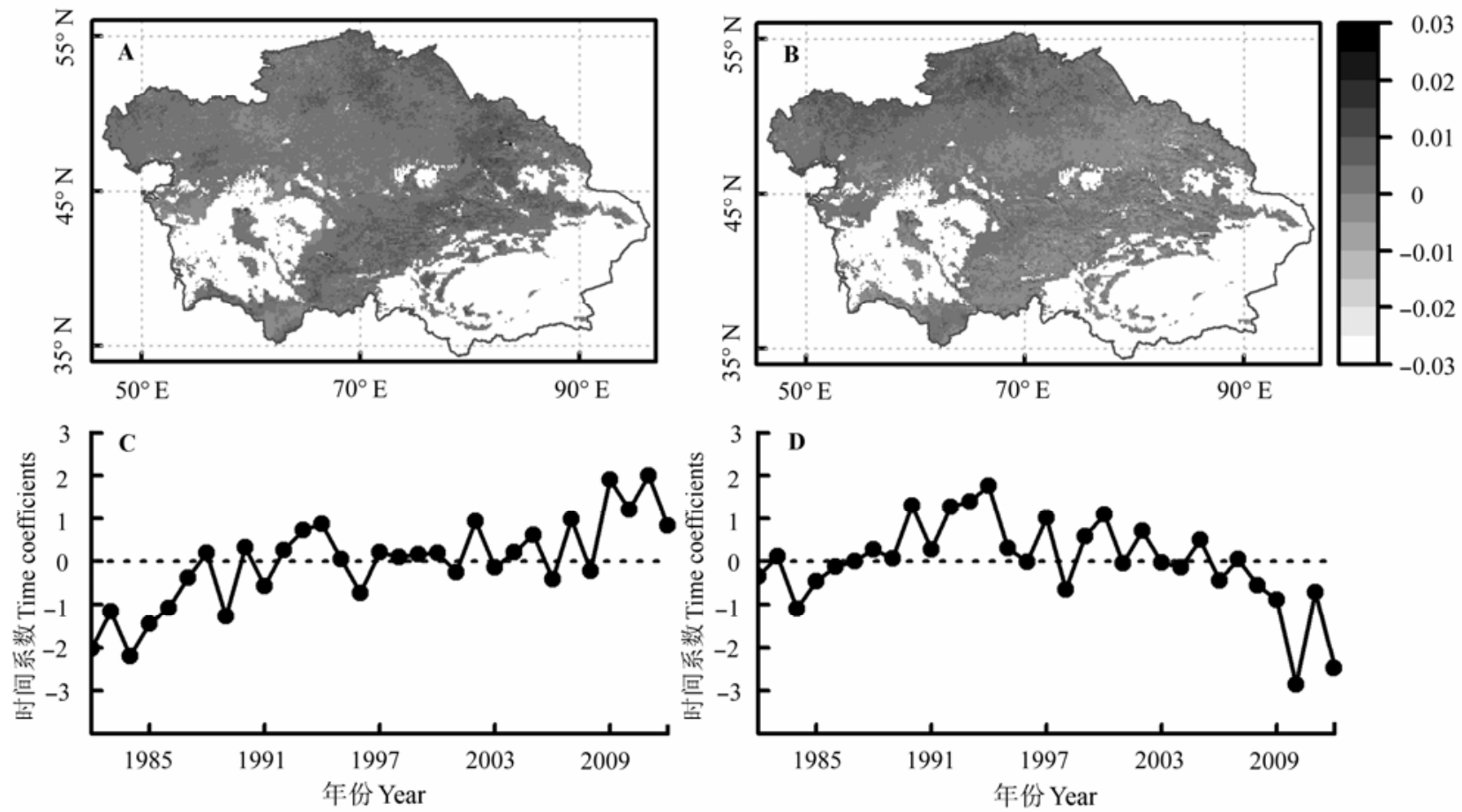

图3 1982-2012年中亚植被年均归一化植被指数(NDVI)经验正交分解的前两个主要空间模态(A、B)及其对应的时间系数(C、 D)。

Fig. 3 The first two leading spatial modes (A, B) and their associated time coefficients (C, D) obtained from empirical orthogonal function of annual mean normalized difference vegetation index (NDVI) over Central Asia in the period of 1982-2012.

表1 中亚植被年均归一化植被指数、年降水量、年平均气温经验正交 分解的前三个空间模态的方差贡献率。

Table 1 The variance contribution by the first three leading modes of annual mean normalized difference vegetation index $(N D V I)$, annual precipitation and annual mean air temperature in Central Asia from empirical orthogonal function.

\begin{tabular}{|c|c|c|c|}
\hline \multirow[t]{2}{*}{$\begin{array}{l}\text { 模态 } \\
\text { Mode }\end{array}$} & \multicolumn{3}{|c|}{$\begin{array}{c}\text { 方差贡献率 } \\
\text { Variance contribution (\%) }\end{array}$} \\
\hline & $\begin{array}{c}\text { 归一化植被指数 } \\
\text { Normalized difference } \\
\text { vegetation index }\end{array}$ & $\begin{array}{c}\text { 降水量 } \\
\text { Precipitation }\end{array}$ & $\begin{array}{c}\text { 气温 } \\
\text { Air temperature }\end{array}$ \\
\hline 1 & 22 & 35 & 74 \\
\hline 2 & 17 & 15 & 10 \\
\hline 3 & 9 & 8 & 6 \\
\hline $\begin{array}{l}\text { 累计方差 } \\
\text { Cumulative } \\
\text { variance }\end{array}$ & 48 & 58 & 90 \\
\hline
\end{tabular}

\subsubsection{NDVI与降水、NDVI与气温的SVD分析}

$N D V I$ 与降水的SVD分析 (图6), 前三组空间模 态的累计方差贡献率为 $76 \%$ (表2)。第一组模态反映 了 $N D V I$ 与降水在空间分布特征上表现较为一致, 变化最显著的区域均位于天山、帕米尔高原; 其时 间系数的相关系数为 $0.73(p<0.01)$ 。这表明天山、 帕米尔高原植被 $N D V I$ 主要受到降水的影响, 与偏 相关分析结果一致(图2A)。第二组模态主要分布于
图尔盖洼地、图尔盖高原, 其时间系数的相关系数 为 $0.81(p<0.01)$, 反映出图尔盖洼地、图尔盖高原 $N D V I$ 变化受到降水变化的影响。在第三组模态中 $N D V I$ 与降水的时间系数的相关系数为 $0.75(p<$ $0.01)$, 也反映出中亚东北部 $N D V I$ 的变化受到降水 的影响。

$N D V I$ 与气温的SVD分析(图7), 前三组空间模态 的累计方差为 $94 \%$ (表 2 )。第一组模态的方差贡献率 为 $77 \%$, 其时间系数的相关系数为 $0.64(p<0.01$, 图 $7 \mathrm{C})$ 。整个中亚气温呈增加趋势(图7B); $N D V I$ 在大部 分地区呈增加趋势, 局部地区呈降低趋势(图7A), 与 线性趋势分析的结果一致(图1D)。该组模态反映了中 亚植被 $N D V I$ 对气温的响应存在较大的空间差异。

\section{3 讨论}

\section{1 植被NDVI在时间和空间上的变化}

$34 \%$ 的中亚植被 $N D V I$ 显著增长，仅有 $4 \%$ 的植 被显著下降, 这与Propastin等(2008a)和索玉霞等 (2009)对中亚 $N D V I$ 变化的研究结果一致。Propastin 等(2008a)发现1982-2003年中亚植被(GIMMS-NDVI) 

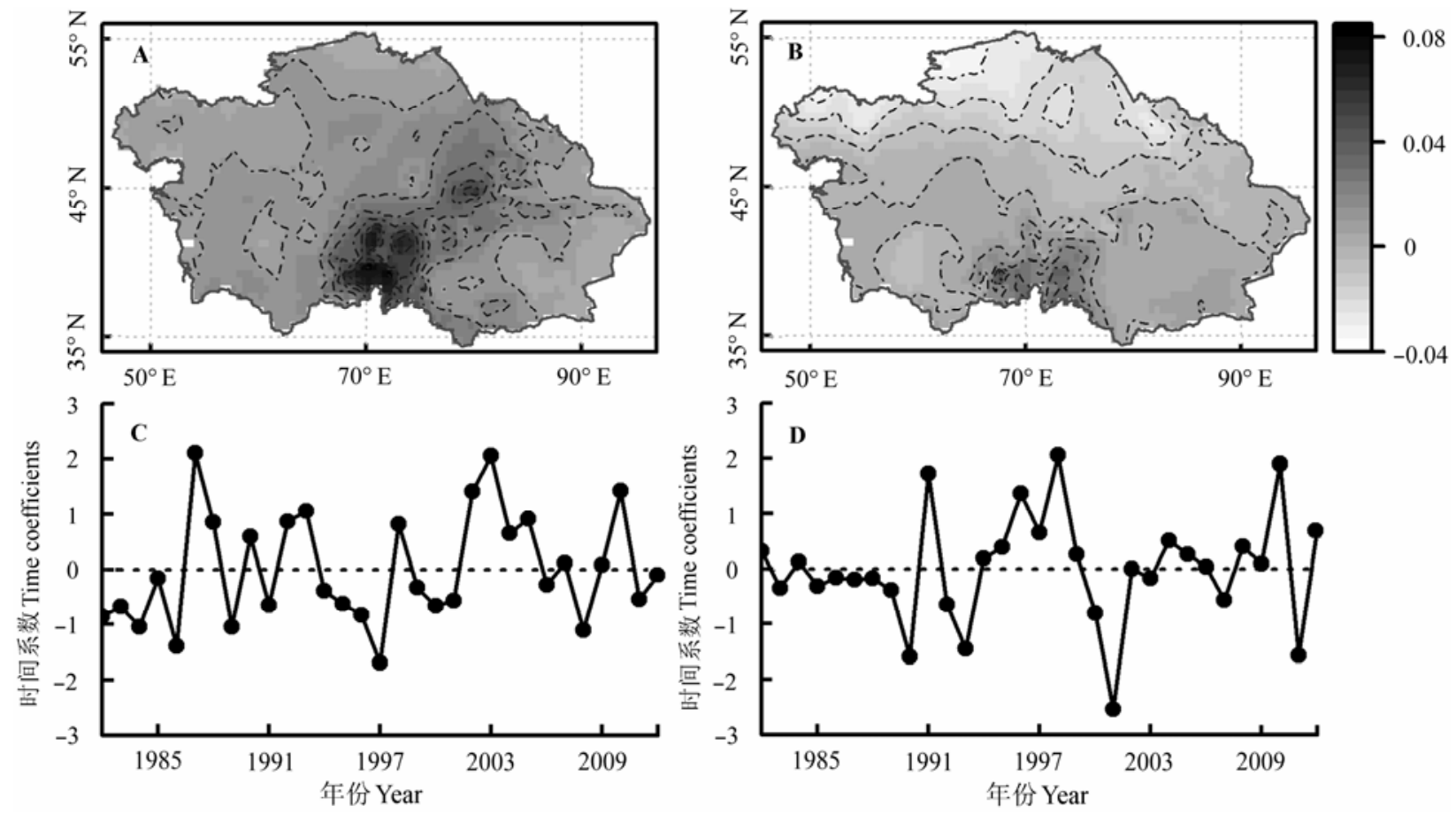

图4 1982-2012年中亚年降水量经验正交分解的前两个主要空间模态(A, B)及其对应的时间系数(C, D)。

Fig. 4 The first two leading spatial patterns (A, B) and their associated time coefficients (C, D) obtained from empirical orthogonal function of annual precipitation over Central Asia in the period of 1982-2012.
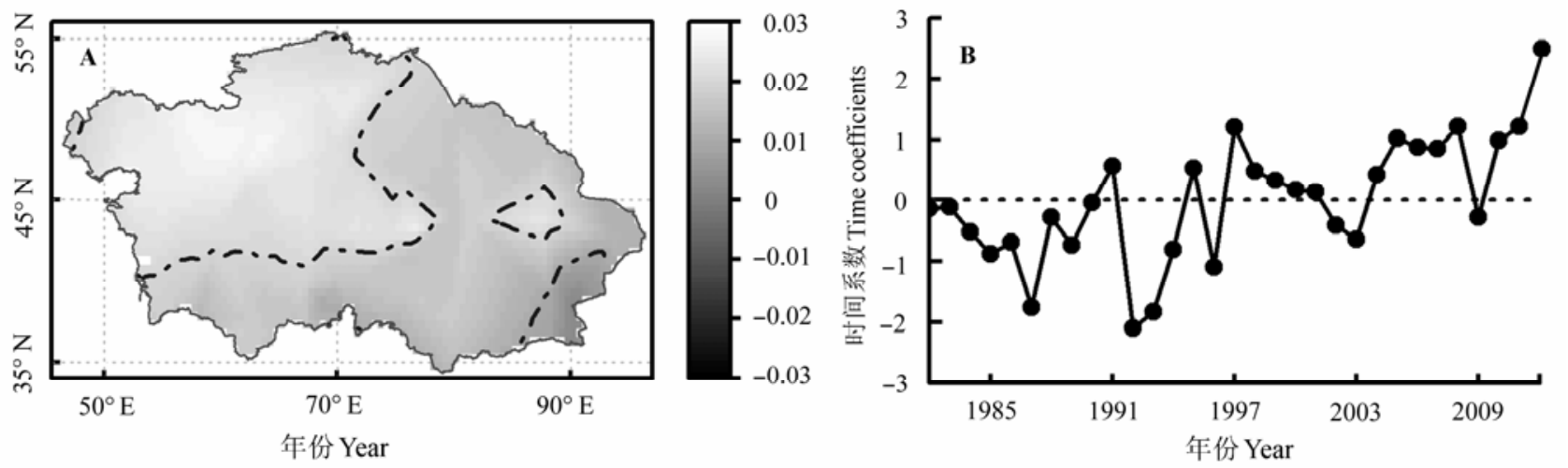

图5 1982-2012年中亚年平均气温经验正交分解的第一个主要空间模态(A)及其对应的时间系数(B)。

Fig. 5 The first leading spatial patterns (A) and its associated time coefficients (B) obtained from empirical orthogonal function of annual mean air temperature over Central Asia in the period of 1982-2012.

总体呈增长趋势, $35 \%$ 的植被显著增长 $\left(R^{2}=0.7\right)$, 仅 2.17\%显著下降。索玉霞等(2009)在1982-2002年的 研究中发现中亚 $39.97 \%$ 的植被(GIMMS-NDVI) 呈增 长趋势, $6.47 \%$ 呈降低趋势。但这种变化趋势与Zhou 等(2015)的研究结果存在差异, 其研究发现整个中 亚区域的植被 $N D V I$ 在1991年之前呈增长的趋势, 在1992年之后呈现相反的减少趋势, 而本研究只发 现中亚的西北部平原区在1994年之后出现减少的趋 势，东部高海拔区域植被 $N D V I$ 呈现增加趋势。

这种研究差异可能与研究区域的范围有关,
Zhou等(2015)的研究范围不包括中国新疆，植被覆 盖区域以哈萨克斯坦北部为主。近几十年新疆由于 降水(尤其是北疆)的增加, 植被 $N D V I$ 显著增加 (Zhao et al., 2011; Du et al., 2015)。本文将新疆加入 中亚研究区使哈萨克斯坦北部植被 $N D V I$ 的变化对 中亚植被 $N D V I$ 变化的贡献程度减少, 导致中亚区 域的植被 $N D V I$ 呈现增长的趋势。并且, $N D V I$ 的 $\mathrm{EOF}$ 分析发现中亚植被变化存在空间差异：东北部及山 区的植被 $N D V I$ 变化主要表现为先增长(1982-1994 年)、后波动(1994-2002年)、再继续增长(2002-2012 

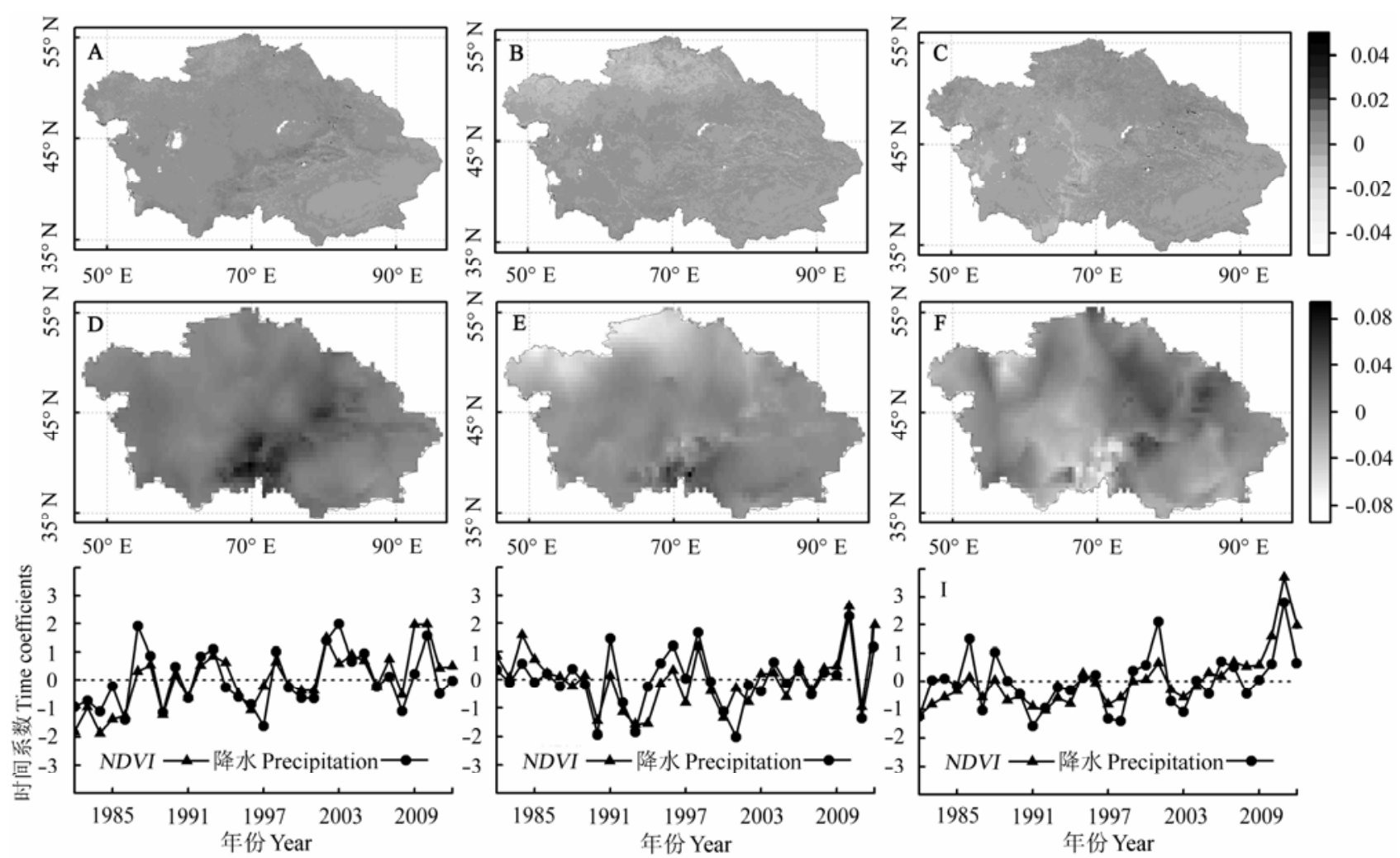

图6 1982-2012年中亚植被年均归一化植被指数 $(N D V I)(\mathbf{A} 、 \mathbf{B} 、 \mathbf{C})$ 和年降水量 $(\mathbf{D} 、 \mathbf{E} 、 \mathbf{F})$ 奇异值分解得到的前 3 个主要特征向 量的空间分布和时间系数 $(\mathbf{G} 、 \mathbf{H} 、 \mathbf{I})$ 。

Fig. 6 Spatial patterns of the first three leading modes obtained from singular value decomposition between normalized difference vegetation index $(N D V I)(\mathbf{A}, \mathbf{B}, \mathbf{C})$ and annual precipitation $(\mathbf{D}, \mathbf{E}, \mathbf{F})$ for the period of 1982 to 2012, and their corresponding time coefficients $(\mathbf{G}, \mathbf{H}, \mathbf{I})$.

表2 中亚植被年均归一化植被指数 $(N D V I)$ 与年降水量、年平均气温奇异值分解的前三个空间模态的方差贡献率。

Table 2 The variance contribution by the first three leading modes of singular value decomposition between annual mean normalized difference vegetation index $(N D V I)$ and annual precipitation, and annual mean air temperature in Central Asia.

\begin{tabular}{|c|c|c|c|c|}
\hline \multirow{2}{*}{$\begin{array}{l}\text { 模态 } \\
\text { Mode }\end{array}$} & \multicolumn{2}{|c|}{$N D V I$ 与降水量 $N D V I$ and precipitation } & \multicolumn{2}{|c|}{$N D V I$ 与气温 $N D V I$ and air temperature } \\
\hline & $\begin{array}{c}\text { 方差贡献率 } \\
\text { Variance contribution (\%) }\end{array}$ & $\begin{array}{c}\text { 相关系数 } \\
\text { Correlation coefficients } \\
\end{array}$ & $\begin{array}{c}\text { 方差贡献率 } \\
\text { Variance contribution (\%) }\end{array}$ & $\begin{array}{c}\text { 相关系数 } \\
\text { Correlation coefficients } \\
\end{array}$ \\
\hline 1 & 49 & $0.73^{*}$ & 77 & $0.64^{*}$ \\
\hline 2 & 19 & $0.81^{*}$ & 13 & -0.38 \\
\hline 3 & 8 & $0.75^{*}$ & 4 & 0.02 \\
\hline
\end{tabular}

*,p<0.01。
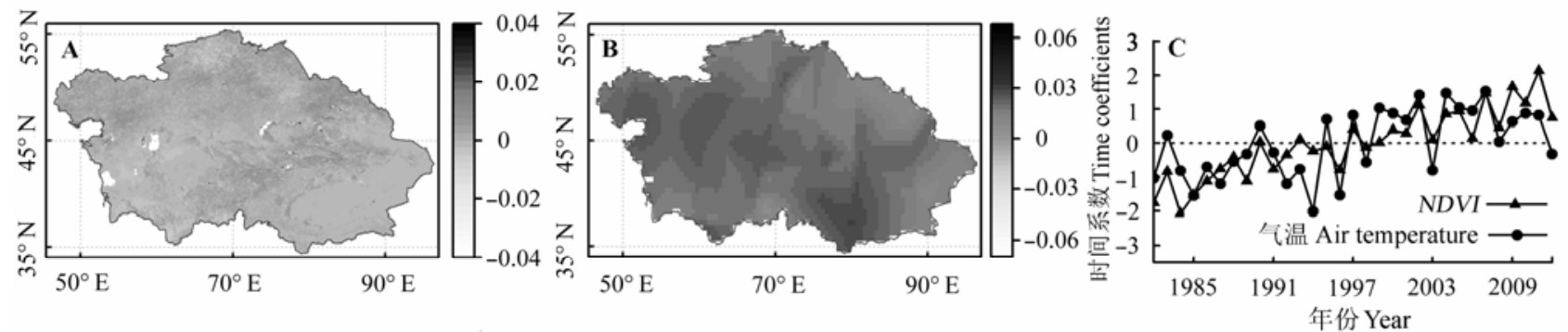

图7 1982-2012年中亚植被归一化植被指数(NDVI)(A)和气温(B)奇异值分解得到的第一个主要特征向量的空间分布和时间系 数 $(\mathbf{C})$ 。

Fig. 7 Spatial patterns of the first leading modes obtained from singular value decomposition between normalized difference vegetation index $(N D V I)(\mathbf{A})$ and annual mean air temperature (B) for the period of 1982 to 2012 and associated time coefficients $(\mathbf{C})$. 
年)的趋势, 而西北部平原区(哈萨克斯坦部分区域) 的植被 $N D V I$ 变化主要表现为先增长(1982-1994年)、 后减少(1994-2012年)的趋势。这种空间差异也会影 响中亚(包括新疆)植被NDVI的整体变化趋势。此外, 研究时段的差异对植被变化的分析结果也会产生影 响。Zhou等 $(2007,2015)$ 对植被NDVI的趋势分析都 是基于时间序列的线性回归分析，由于这种方法依 赖于研究时段内植被 $N D V I$ 的平均值, 研究时间长 度的差异会直接影响植被的变化趋势。

\section{2 植被变化对气候因子的响应}

本研究利用偏相关和SVD两种方法分析了植被 NDVI对气象因子(气温和降水)的响应关系。分析结 果显示, $63 \%$ 的植被 $N D V I$ 与降水显著相关, $32 \%$ 与气 温显著相关。植被对降水的响应在空间上较为均一, 主要表现为正相关。而植被对气温的响应存在空间 异质性, $23 \%$ 与气温呈显著负相关, 分布在西北部, $9 \%$ 与气温呈显著正相关, 主要分布在东南部海拔 较高的山区, 该区域的降水量高于西北部(Chen et al., 2011)。Mohammat等(2013)研究发现在高海拔区 域, 植被生长主要受限于气温, 这与本文研究结果 一致。原因可能是高海拔区域气温低(Hu et al., 2014), 植被蒸腾耗水量少, 植被生长对降水的依赖 减弱。大量研究表明, 在过去几十年, 由于全球变 暖、 $\mathrm{CO}_{2}$ 浓度升高, 北半球植被活动显著增强(方精 云等, 2003)。然而, 植被活动自20世纪末以来有所 减弱甚至出现降低的趋势。Piao等(2011)研究发现在 欧亚大陆温带以北, 植被 $N D V I$ 在1997年之后出现 减少的趋势, 主要是由于降水的减少引起的。Peng 等(2011)也发现, 中国植被活动在 20 世纪 90 年代之 后也出现减弱的趋势, 主要是由于气温的升高造成 干旱程度的加剧所引起的。本文的研究结果也表明: 1994年之后在中亚西北部植被 $N D V I$ 出现减少的趋 势, 但由于降水并未出现明显的变化趋势, 所以植 被 $N D V I$ 的下降可能是气温升高造成的。

传统的相关分析在解释变量间的关系时存在一 定的局限性。首先, 相关分析无法说明变量间的关 系是否存在一定的偶然性, 这种偶然性可能是由于 变量存在的共同趋势、周期等现象产生的。再者, 相 关分析是否显著也无法解释变量间是否存在因果关 系(Gimeno et al., 1999)。本文关于气温变化与植被 $N D V I$ 变化的相关关系研究, 尽管对气候变量和植 被 $N D V I$ 都进行了距平处理以及趋势和周期性检验,
但仍然无法确定气候变量与植被 NDVI之间的相关 关系是否是假相关。基于变量之间的因果关系检测, 包括收敛交叉映射法(CCM) (Sugihara et al., 2012), 格兰杰因果检验(GC) (Jiang et al., 2015)或者其他类 似的统计方法, 将有助于分析气候对植被变化的影 响(Jiang et al., 2015; Tsonis et al., 2015), 从而辨别 变量之间是否存在假相关。然而, 所有这些统计方 法都在很大程度上受限于研究区域内气候和植被变 化幅度以及研究时段时间长短的影响。

\section{3 不确定性分析}

气候变量的不确定性也会对本文的研究结果产 生影响。基于不同的数据集, 近 30 年中亚降水的变 化趋势存在较大的差异。基于全球陆面数据同化系 统(GLDAS)的数据发现中亚降水明显减少(Kariyeva et al., 2012)。然而, 基于CRU数据发现中亚的降水 是在增加的(Chen et al., 2011)。这种相反的降水变化 趋势可能是气象数据本身的不确定性引起的。本研 究所采用的 $\mathrm{CRU}$ 数据集是由全球气象站点插值形 成的, 但这些站点的分布并不均匀, 特别是在中亚, 苏联解体以后大量气象站被废弃(Hu et al., 2014)。 因此, 20 世纪 90 年代之后中亚降水是否减少还需要 进一步研究。中亚植被变化不仅受到气温、降水等 自然因素的影响, 也受到人类活动的影响, 尤其是 土地利用/土地覆盖变化(de Beurs \& Henebry, 2004)。20世纪90年代以前, 中亚哈萨克斯坦大量的 土地被开垦(Zhou et al., 2015); 1991年，前苏联解体， 大量农田被弃耕, 畜牧量急剧下降(Propastin et al., 2008a), 对植被的类型分布、覆盖产生了明显的影 响。此外, 2001-2009年全球火灾研究显示中亚哈萨 克斯坦草地是全球主要的受灾区域之一(Loboda et $a l ., 2012)$, 显著影响了中亚植被 $N D V I$ 的变化。同时, 放牧、城市化等人类活动, 对中亚植被 $N D V I$ 变化也 有较大的影响作用(Luo et al., 2012)。

\section{4 结论}

本文基于线性趋势分析、偏相关分析、经验正 交分解、奇异值分解，详细探讨了1982-2012年中亚 植被变化特征, 并结合降水、气温数据, 从空间和时 间两个维度上分析了中亚植被对气候因子的响应关 系, 得到以下结论:

(1) 1982-2012年, 中亚植被活动增强, 在山区 更为明显。中亚植被 $N D V I$ 的变化在空间上表现出较 
大的异质性。中亚山区及东北部的植被 $N D V I$ 变化主 要表现为先增长(1982-1994年)、后波动(1994-2002 年)、再继续增长(2002-2012年)的趋势。而中亚西北 部平原区的植被 $N D V I$ 变化主要表现为先增长 (1982-1994年)、后减少(1994-2012年)的趋势。

(2) 中亚植被活动受降水和气温的共同影响。 降水对植被活动的影响更加明显, 特别是在中亚西 北部的平原区域。而气温对植被活动的影响主要体 现在东部海拔较高的区域。

(3) 自 20 世纪 90 年代初以来, 在中亚西北部平 原区域, 气温的增加、降水的减少导致了植被 $N D V I$ 呈下降趋势。而在东部高海拔山区, 由于降水相对 充沛以及气温的升高, 促进了植被生长, 植被 $N D V I$ 呈现出显著增加趋势。

EOF和SVD相结合为研究植被变化及植被与气 候变量之间的关系提供了一种基于空间和时间两个 维度的分析方法, 在分析区域植被变化的时空特征 中具有较好的适用性。然而, 植被NDVI对气候的响 应具有较大的时空差异, 不同类型的植被 NDVI对 气候变化的响应也存在着较大差异。本研究从整个 中亚区域和年际尺度上分析了植被NDVI对降水和 气温变化的响应, 今后的工作将进一步分析中亚不 同植被类型、不同区域以及季节尺度上的 $N D V I$ 变化 及其对气候变化的响应关系。

基金项目 国家自然科学基金委员会一新疆联合基 金“本地优秀青年人才培养专项” (U1403382)，国家 自然科学基金“面上项目”(41171094)。

\section{参考文献}

Bojanowski JS, Kowalik W, Bochenek Z (2009). Noise reduction of NDVI time series: A robust method based on Savitzky-Golay filter. Annals of Geomatics, 7, 13-21.

Chen FH, Huang W, Jin LY, Chen JH, Wang JS (2011). Spatiotemporal precipitation variations in the arid Central Asia in the context of global warming. Science China Earth Sciences, 54, 1812-1821.

Chen X (2012). Retrieval and Analysis of Evapotranspiration in Central Areas of Asia. China Meteorological Press, Beijing. 111. (in Chinese) [陈䂀 (2012). 亚洲中部干旱区蒸 散发研究. 气象出版社, 北京. 111.]

de Beurs KM, Henebry GM (2004). Land surface phenology, climatic variation, and institutional change: Analyzing agricultural land cover change in Kazakhstan. Remote Sensing of Environment, 89, 497-509.

de Beurs KM, Wright CK, Henebry GM (2009). Dual scale trend analysis for evaluating climatic and anthropogenic effects on the vegetated land surface in Russia and Kazakhstan. Environmental Research Letters, 4, 940-941. doi: 10.1016/ j.rse.2003.11.006.

Du JQ, Shu JM, Yin JQ, Yuan XJ, Jiaerheng A, Xiong SS, He P, Liu WL (2015). Analysis on spatio-temporal trends and drivers in vegetation growth during recent decades in Xinjiang, China. International Journal of Applied Earth Observation and Geoinformation, 38, 216-228.

Fang JY, Piao SL, He JS, Ma WH (2003). Vegetation activity increased in China in nearly 20 years. Science in China Series C: Life Sciences, 33, 554-565. (in Chinese) [方精 云, 朴世龙, 贺金生, 马文红 (2003). 近20年来中国植 被活动在增强. 中国科学(C辑:生命科学), 33, 554-565.]

Fensholt R, Langanke T, Rasmussen K, Reenberg A, Prince SD, Tucker C, Scholes RJ, Le QB, Bondeau A, Eastman R, Epstein H, Gaughan AE, Hellden U, Mbow C, Olsson L, Paruelo J, Schweitzer C, Seaquist J, Wessels K (2012). Greenness in semi-arid areas across the globe 1981-2007: An earth observing satellite based analysis of trends and drivers. Remote Sensing of Environment, 121, 144-158.

Gessner U, Naeimi V, Klein I, Kuenzer C, Klein D, Dech S (2013). The relationship between precipitation anomalies and satellite-derived vegetation activity in Central Asia. Global and Planetary Change, 110, 74-87.

Gimeno R, Manchado B, Mínguez R (1999). Stationarity tests for financial time series. Physica A: Statistical Mechanics and its Applications, 269, 72-78.

Hu ZY, Zhang C, Hu Q, Tian HQ (2014). Temperature changes in Central Asia from 1979 to 2011 based on multiple datasets. Journal of Climate, 27, 1143-1167.

IGBP (The International Geosphere Biosphere Programme) (1997). The Terrestrial Biosphere and Global Change. Cambridge University Press, Stockholm, Sweden.

Jiang B, Liang SL, Yuan WP (2015). Observational evidence for impacts of vegetation change on local surface climate over northern China using the Granger causality test. Journal of Geophysical Research, 120, 1-12.

Johnson RA, Wichern DW (2007). Applied Multivariate Statistical Analysis. 6th edn. Pearson Prentice Hall Press, US. 401-409.

Jordi A, Basterretxea G (2012). Using SVD analysis of combined altimetry and ocean color satellite data for assessing basin scale physical-biological coupling in the Mediterranean Sea. Remote Sensing of Biomass, 6, 123-140.

Kariyeva J, van Leeuwen WJD, Woodhouse CA (2012). Impacts of climate gradients on the vegetation phenology of major land use types in Central Asia (1981-2008). Frontiers of Earth Science, 6, 206-225.

Lioubimtseva E, Cole R, Adams JM, Kapustin G (2005). Impacts of climate and land-cover changes in arid lands of Central Asia. Journal of Arid Environments, 62, 285-308.

Lioubimtseva E, Henebry GM (2009). Climate and environmental change in arid Central Asia: Impacts, vulnerability,

www.plant-ecology.com 
and adaptations. Journal of Arid Environments, 73, 963977.

Loboda TV, Giglio L, Boschetti L, Justice CO (2012). Regional fire monitoring and characterization using global NASA MODIS fire products in dry lands of Central Asia. Frontiers of Earth Science, 6, 196-205.

Luo GP, Han QF, Zhou DC, Li L, Chen X, Li Y, Hu YK, Li BL (2012). Moderate grazing can promote aboveground primary production of grassland under water stress. Ecological Complexity, 11, 126-136.

Mohammat A, Wang XH, Xu XT, Peng LQ, Yang Y, Zhang XP, Myneni RB, Piao SL (2013). Drought and spring cooling induced recent decrease in vegetation growth in Inner Asia. Agricultural and Forest Meteorology, 178-179, 21-30.

Park HS, Sohn BJ (2010). Recent trends in changes of vegetation over East Asia coupled with temperature and rainfall variations. Journal of Geophysical Research, 115, D14101. doi: 10.1029/2009JD012752.

Peng SS, Chen AP, Xu L, Cao CX, Fang JY, Myneni RB, Pinzon JE, Tucker CJ, Piao SL (2011). Recent change of vegetation growth trend in China. Environmental Research Letters, 6, 044027. doi: 10.1088/1748-9326/6/4/044027.

Piao SL, Wang XH, Ciais P, Zhu B, Wang T, Liu J (2011). Changes in satellite-derived vegetation growth trend in temperate and boreal Eurasia from 1982 to 2006. Global Change Biology, 17, 3228-3239.

Prohaska JT (1976). A technique for analyzing the linear relationships between two meteorological fields. Monthly Weather Review, 104, 1345-1353.

Propastin PA, Kappas M, Muratova NR (2008a). Inter-annual changes in vegetation activities and their relationship to temperature and precipitation in Central Asia from 1982 to 2003. Journal of Environmental Informatics, 12, 75-87.

Propastin PA, Kappas M, Muratova NR (2008b). A remote sensing based monitoring system for discrimination between climate and human-induced vegetation change in Central Asia. Management of Environmental Quality, 19, 579-596.

Slayback DA, Pinzon JE, Los SO, Tucker CJ (2003). Northern hemisphere photosynthetic trends 1982-99. Global Change Biology, 9, 1-15.

Sugihara G, May R, Ye H, Hsieh CH, Deyle E, Fogarty M, Munch S (2012). Detecting causality in complex ecosystems. Science, 338, 496-500.

Suo YX, Wang ZX, Liu C, Yu BH (2009). Relationship between NDVI and precipitation and temperature in Middle Asia during 1982-2002. Resources Science, 31, 1422 1429. (in Chinese with English abstract) [索玉霞, 王正兴, 刘闯，于伯华 (2009). 中亚地区1982年至2002年植被指 数与气温和降水的相关性分析. 资源科学, 31，14221429.]

Tsonis AA, Deyle ER, May RM, Sugihara G, Swanson K, Verbeten JD, Wang GL (2015). Dynamical evidence for causality between galactic cosmic rays and interannual variation in global temperature. Proceedings of the $\mathrm{Na}$ tional Academy of Sciences of the United States of America, 112, 3253-3256.

Wei FY (2007). Modern Climate Statistics and Forecast Technology. 2nd edn. China Meteorological Press, Beijing. 114. (in Chinese) [魏凤英 (2007). 现代气候统计诊断与 预测技术 第二版. 气象出版社, 北京. 114.]

Wright CK, de Beurs KM, Akhmadieva ZK, Groisman PY, Henebry GM (2009). Reanalysis data underestimate significant changes in growing season weather in Kazakhstan. Environmental Research Letters, 4, 0450202. doi: 10. 1088/1748- 9326/4/4/045020.

Xu XR (2005). Remote Sensing Physics. The Peking University Publishing House, Beijing. 176. (in Chinese) [徐希濡 (2005). 遥感物理. 北京大学出版社, 北京. 176.]

Zhang XZ (2014). Main models of variations of autumn vegetation greenness in the mid-latitude of north hemisphere in 1982-2011. Scientia Geographica Sinica, 34, 1226-1232. (in Chinese with English abstract) [张学珍 (2014). 1982-2011年北半球中纬度秋季植被绿度变化的主要模 态. 地理科学, 34, 1226-1232.]

Zhao X, Tan K, Zhao SQ, Fang JY (2011). Changing climate affects vegetation growth in the arid region of the northwestern China. Journal of Arid Environments, 75, 946952.

Zhou KF, Zhang Q, Chen X, Sun L (2007). Features and trends of the environmental change in the arid areas in Central Asia. Science China Series D: Earth Sciences, 50, 142-148.

Zhou Y, Zhang L, Fensholt R, Wang K, Vitkovskaya I, Tian F (2015). Climate contributions to vegetation variations in Central Asian drylands: Pre-and Post-USSR collapse. Remote Sensing, 7, 2449-2470.

责任编委: 唐志尧 责任编辑: 王 葳 


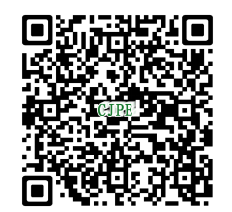

植物生态学报官网

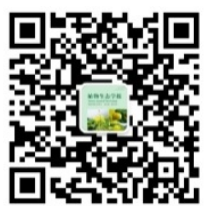

微信订阅号

期刊及学科

相关信息发布

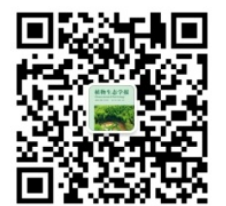

微信服务号

稿件状态查询

全文检索汶览 